\title{
Regulated Breathless receptor tyrosine kinase activity required to pattern cell migration and branching in the Drosophila tracheal system
}

\author{
Tzumin Lee, ${ }^{1}$ Nir Hacohen, ${ }^{2}$ Mark Krasnow, ${ }^{2}$ and Denise J. Montell ${ }^{1,3}$ \\ ${ }^{1}$ Department of Biological Chemistry, Johns Hopkins School of Medicine, Baltimore, Maryland 21205-2185 USA; \\ ${ }^{2}$ Department of Biochemistry, Stanford University School of Medicine, Stanford, California 94327 USA
}

\begin{abstract}
Receptor tyrosine kinases (RTKs) are members of a diverse class of signaling molecules well known for their roles in cell fate specification, cell differentiation, and oncogenic transformation. Recently several RTKs have been implicated in cell and axon motility, and RTKs are known to mediate chemotactic guidance of tissue culture cells. We have investigated whether the Drosophila FGF receptor homolog, Breathless (BTL), whose activity is necessary for each phase of branching morphogenesis in the embryonic tracheal system, might play a role in guiding the directed migration of tracheal cells. We found that expression of a constitutively active receptor during tracheal development interfered with directed tracheal cell migration and led to extra secondary and terminal branch-forming cells. Reduction in endogenous BTL signaling enhanced the cell migration defects while suppressing the ectopic branching defects. These results are consistent with a model for tracheal development in which spatially regulated BTL activity guides tracheal cell migration and quantitatively regulated BTL activity determines the patterns of secondary and terminal branching cell fates.
\end{abstract}

[Key Words: Cell migration; branching morphogenesis; receptor tyrosine kinase]

Received June 5, 1996; revised version accepted September 24, 1996.

Morphogenesis of branched tubular networks is an essential element of the normal development of several organ systems, including the lungs and vasculature. Formation of branched tubular structures involves determination of multiple cell fates, cell migration, and extensive cell shape changes. The mechanisms by which these diverse biological processes are coordinated to establish a complicated network remain a puzzle.

The Drosophila tracheal (respiratory) system is a network of branched tubules that ramify extensively through the body, allowing oxygen diffusion to all tissues. The embryonic tracheal system originates from 10 pairs of tracheal placodes, each of which invaginates to form a sac of about 80 tracheal cells. The tracheal cells then migrate along stereotyped pathways, and some branches meet and fuse to form the primary pattern of tracheal branches (Manning and Krasnow 1993; Samakovlis et al. 1996). Subsequently, selected tracheal cells generate secondary branches. Each secondary branch is formed by a single tracheal cell and extends in a stereotypical direction. Finally, terminal branches are formed as subcellular tubes within long cytoplasmic processes. Terminal branches originate from most secondary

${ }^{3}$ Corresponding author. branch-forming cells and their extensions are believed to be regulated by target tissue oxygen need. A gene regulatory hierarchy controls the different stages of branching, and a number of enhancer trap lines have been described that mark specific subsets of tracheal cells, such as secondary or terminal branch-forming cells (Samakovlis et al. 1996). However, the spatial cues that select these cell fates are not known.

Specific receptor tyrosine kinases (RTKs) have been found to play a role in morphogenesis of tubular networks in a variety of organisms. In particular, fibroblast growth factors (FGF) and their receptors are potent angiogenic factors in mammals, and Breathless (BTL), a Drosophila FGF receptor homolog, the product of the breathless (btl) gene, is known to be required for proper tracheal morphogenesis (Glazer and Shilo 1991; Klambt et al. 1992; Reichman-Fried et al. 1994). Furthermore, RTKs are well known for their ability to control cell fate, cell differentiation programs, and cell motility (Basler et al. 1991; Montell 1994; Obermeier et al. 1994; Schuchardt et al. 1994b; Bladt et al. 1995; DeVore et al. 1995). Thus RTKs are candidates for controlling many aspects of branching morphogenesis.

The breathless gene was cloned by low-stringency hybridization with a vertebrate FGF receptor probe and was found to be expressed specifically in tracheal cells, mid- 
line glial cells, and salivary duct cells of the Drosophila embryo (Glazer and Shilo 1991). In btl mutant embryos, tracheal cells fail to migrate out from the tracheal sacs, and hence the embryos display severe tracheal system defects (Klambt et al. 1992). The number of tracheal cells and the formation of tracheal sacs are not affected in $b t l$ loss-of-function mutants, suggesting that the BTL RTK is specifically required for subsequent morphogenetic events. BTL activity is also required for formation of secondary and terminal branches (Reichman-Fried and Shilo 1995). However, the precise role of breathless in tracheal morphogenesis is not clear. For example, it is not known whether BTL activity primarily determines various cell fates within the tracheal system, promotes general cell motility, or plays a chemotactic guidance role in tracheal cell migration.

It has been shown previously that constitutively active Sevenless (SEV) functions effectively in cell fate determination (Dickson et al. 1996). However, a chemotactic guidance role for a receptor would, by definition, require different levels of receptor activity in different parts of each tracheal cell. We found that a constitutively active BTL receptor inhibited primary branch outgrowth but promoted ectopic secondary and terminal branch formation. Our results support the hypothesis that regulated BTL activity guides tracheal cell migration and determines the pattern of secondary and terminal branches by selecting tracheal cell fates. Thus regulation of BTL activity is key to each phase of tracheal morphogenesis.

\section{Results}

\section{$\lambda B T L$, a putative constitutively active RTK}

Activation of RTKs typically depends on ligand-induced dimerization followed by autophosphorylation (Fantl et al. 1993). Therefore replacement of the ligand-binding extracellular domain of a receptor with a protein domain that spontaneously dimerizes might be predicted to create a constitutively active receptor. This rationale was employed previously to create a constitutively active $X e$ nopus FGFR chimera, composed of the dimerization domain of $\lambda$ repressor and the transmembrane and cytoplasmic domains of Xenopus FGFR (E. Amaya, pers. comm.). We used the same strategy to make a putative constitutively active lambda-BTL chimera $(\lambda \mathrm{BTL}$, Fig. 1A), and transgenic fly strains carrying the $\lambda b t 1$ transgene under the control of the hsp 70 heat-inducible promoter and the GAL4-inducible UAS promoter (Brand and Perrimon 1994) were generated.

To determine whether $\lambda$ BTL was constitutively autophosphorylated on tyrosine, immunoprecipitation (IP) and immunoblotting studies were performed. After expressing $\lambda$ BTL in embryos by heat shock, embryo lysates were immunoprecipitated with either anti-BTL or antiphosphotyrosine (pY) antibodies, and then the immune complexes were analyzed by PAGE followed by Western blotting with either anti-BTL or anti-pY antibodies. The $\lambda \mathrm{BTL}$ protein was immunoprecipitated by anti-pY Ab as shown in Figure $1 B$, lane 7 . In addition, the $\lambda B T L$ protein immunoprecipitated with the anti-BTL Ab was recog-
A

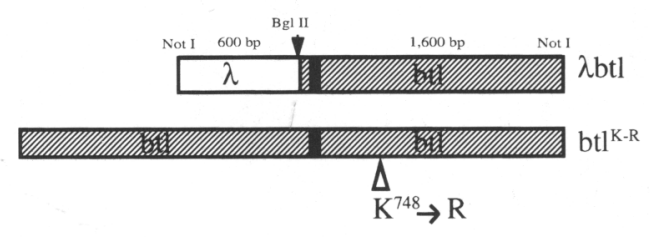

B

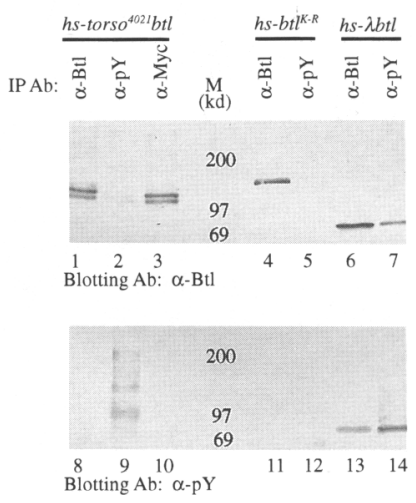

Figure 1. Constitutively active and kinase-defective BTL constructs. $|A|$ Schematic drawing of the $\lambda$ BTL chimera and $\mathrm{BTL}^{\mathrm{K} \rightarrow \mathrm{R}}$. $\lambda \mathrm{BTL}$ was constructed by replacing the extracellular domain of BTL with the dimerization domain of $\lambda \mathrm{c} 1$ repressor. $\mathrm{BTL}^{\mathrm{K} \rightarrow \mathrm{R}}$ was constructed by mutating the conserved lysine within the ATP binding domain to arginine. $(B)$ Detection of BTL protein and phosphotyrosine residues in $\mathrm{ATTL}$, Torso ${ }^{4021}$ $\mathrm{BTL}$, and $\mathrm{BTL}^{\mathrm{K} \rightarrow \mathrm{R}}$. After heat shock-induced expression of the BTL transgenes in embryos, lysates were immunoprecipitated with $\alpha$-BTL (lanes 1,4,6,8,11,13), $\alpha$-pY (lanes 2,5,7,9,12,14), or $\alpha-M Y C$, which detects the MYC-tagged Tor ${ }^{4021}$-BTL protein (lanes 3,10). The immune complexes were analyzed by SDSPAGE and Western blotting with $\alpha$-BTL Ab (lanes 1-7) or $\alpha$-pY Ab (lanes 8-14).

nized by the anti-pY Ab in a Western blot (Fig. 1B, lane 13). These results showed that $\lambda B T L$, which lacks the ligand-binding domain, could undergo ligand-independent autophosphorylation, as expected of a constitutively active RTK. In contrast, less phosphotyrosine was detected using the same analysis to examine Torso ${ }^{4021}$ BTL (Fig. 1B, lane 2), a putative constitutively active BTL receptor, reported previously (ReichmanFried et al. 1994). These results suggested that the $\lambda B T L$ chimera provided more activated BTL kinase activity than Torso ${ }^{4021}$ BTL.

As a negative control, we examined the phosphorylation state of a putative kinase-defective BTL RTK $\left(\mathrm{BTL}^{\mathrm{K} \rightarrow \mathrm{R}}\right)$, which was made by mutating the conserved lysine residue within the ATP binding domain to arginine (Fig. 1A). Transgenic fly strains carrying the $b t 1^{K \rightarrow R}$ transgene under heat shock promoter control were generated, and immunoprecipitation followed by Western blot analysis revealed that $\mathrm{BTL}^{\mathrm{K} \rightarrow \mathrm{R}}$ protein was expressed stably following heat shock; however, as expected, no phosphotyrosine could be detected (Fig. 1B, lane 5). 


\section{Effects of $\lambda B T L$ in btl mutant embryos}

A constitutively activated receptor would be expected to substitute effectively for the wild-type receptor if its role were to affect cell fate determination or cell differentiation, as is true for the SEV RTK (Dickson et al. 1996). Thus, although the pattern of activation of this receptor is important because the constitutively active receptor alters the fates of neighboring cells, the activated form functions properly in the cells in which it is normally active. However, if the BTL receptor served a chemotactic guidance function, one would expect the constitutively active receptor not to provide the necessary spatial information and not to substitute effectively for the wild-type receptor in tracheal cells (see, e.g., Kundras et al. 1994).

To determine whether regulated BTL activity was essential for migration of tracheal cells away from the tracheal sacs, we compared the ability of $\lambda$ BTL and wildtype BTL to rescue btl mutant embryos. The tracheal system was visualized by staining the tracheal lumen with the monoclonal antibody $(\mathrm{mAb}) 2 \mathrm{~A} 12$. Figure $2 \mathrm{~A}$ shows the wild-type pattern of tracheal tubules, which are lined by the tracheal cells. In $b t t^{H 82 \Delta 3}$ mutant embryos, tracheal cells fail to migrate, remaining in a tra-
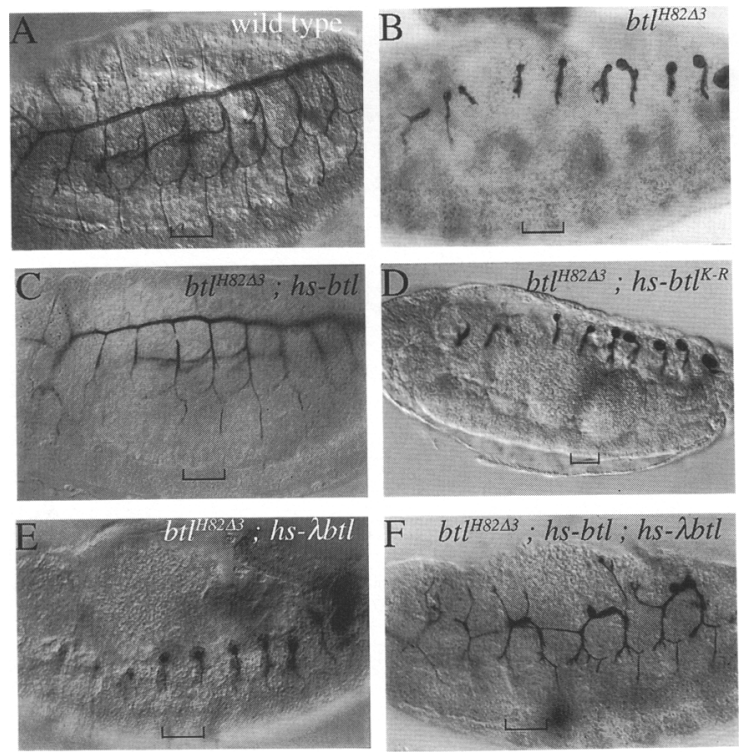

Figure 2. Rescue of tracheal cell migration defects in btl mutant embryos by various btl transgenes. Nomarski optics images of embryos stained for tracheal lumen antigen $2 \mathrm{~A} 12$, to illustrate the extent of tracheal cell migration in embryos of the following genotypes: $(A)$ wild type; $(B)$ homozygous $b t 1^{H 8243}$ mutant; $(C) b t l^{H 82 \Delta 3} / b t l^{H 82 \Delta 3} ; h s-b t l_{;}(D) b t l^{H 82 \Delta 3} / b t l^{H 82 \Delta 3} ; h s$ $b t l^{K \rightarrow R} ;(E) b t l^{H 82 \Delta 3} / b t l^{H 82 \Delta 3} ; h s-\lambda b t l^{\prime}$ and $(F) b t l^{H 82 \Delta 3} / b t I^{H 82 \Delta 3}$; $h s-\lambda b t l ; h s-\lambda b t l$. Embryos were heat shocked for $1 \mathrm{hr}$ at stage 11 , incubated at $18^{\circ} \mathrm{C}$, and fixed at stage 15 . The normal pattern of primary branches can be seen in $A$ and $C$. To determine genotypes, embryos were also stained with an anti- $\beta$-galactosidase antibody, followed by a fluorescently labeled secondary antibody, to detect $\beta$-galactosidase expressed from a transgene on the TM3 balancer chromosome. Bars, $25 \mu \mathrm{m}$. cheal sac-like formation (Klambt et al. 1992). Despite this, the cells still make a lumen and express the luminal antigen detected by mAb2A12 (Fig. 2B). After introducing the wild-type btl transgene under heat shock promoter control into the $b t l$ mutant background, we found that a single heat shock delivered at $4-5 \mathrm{hr}$ of development rescued tracheal cell migration defects and restored the primary branching pattern (Fig. 2C; Table 1). In contrast, heat shock-induced expression of either $\mathrm{BTL}^{\mathrm{K} \rightarrow \mathrm{R}}$ or $\mathrm{ABTL}$ failed to rescue tracheal cell migration defects in stage $15 \mathrm{btl}$ mutant embryos (Fig. 2D and $\mathrm{E}$; Table 1). When the hs- $\lambda$ BTL embryos were allowed to develop to stage 16 or 17 ( $16 \mathrm{hr}$ after egg laying), fusion of the dorsal trunk was observed in two to three posterior segments of approximately half of the embryos (Table 1). Thus constitutively active BTL could rescue only a small fraction of the tracheal pattern, and this was significantly delayed.

If regulated BTL activity were essential to the normal pattern of tracheal cell migration, $\lambda B T L$ might partially obscure the normal pattern of receptor activity, even in the presence of the wild-type receptor, and thereby interfere with the normal migration pattern. To test this possibility, we determined the ability of $h s-b t l$ to rescue $b t l$ mutant embryos when coexpressed with $\lambda B$ BTL. The rescue was less complete than that provided by $h s-b t l$ alone (Fig. 2F; Table 1). Taken together these results indicated that spatially or quantitatively regulated BTL activity was required for the normal pattern of primary branching.

Disruption of wild-type tracheal system development by $\lambda B T L$

If BTL were normally activated in a spatially restricted pattern, expression of a constitutively active BTL recep-

Table 1. Quantitative analysis of dorsal trunk defects

\begin{tabular}{|c|c|c|}
\hline Genotype & $\begin{array}{l}\text { Average no. } \\
\text { of dorsal } \\
\text { trunk } \\
\text { interruptions } \\
\text { per embryo }\end{array}$ & $\begin{array}{l}\text { Embryos with } \\
\text { dorsal trunk } \\
\text { interruptions } \\
(\%)\end{array}$ \\
\hline$+/+($ stage $14 / 15)$ & 0 & 0 \\
\hline$b t l l+$ & 0 & 0 \\
\hline$b t l / b t l$ & 9 & 100 \\
\hline btl/btl;hs-btl (stage 14/15) & 0 & 0 \\
\hline$b t l / b t l ; h s-\lambda b t l$ (stage $14 / 15$ ) & 9 & 100 \\
\hline$b t l / b t l ; h s-\lambda b t l$ (stage $16 / 17$ ) & 7 & 100 \\
\hline \multicolumn{3}{|l|}{$b t 1 / b t l ; h s-b t l ; h s-\lambda b t l$} \\
\hline |stage $14 / 15 \mid$ & 5 & 90 \\
\hline$+/+; h s-\lambda b t l$ (stage $14 / 15$ ) & 2.5 & 50 \\
\hline$b t l+; h s-\lambda b t l$ (stage $14 / 15$ ) & 4 & 70 \\
\hline \multicolumn{3}{|l|}{$+/+; h s-\lambda b t l ; h s-b t I^{K \rightarrow R}$} \\
\hline |stage $14 / 15 \mid$ & 8 & 90 \\
\hline$+/+; h s-b t I^{K \rightarrow R}$ (stage $14 / 15$ ) & 3 & 60 \\
\hline
\end{tabular}

${ }^{a}$ The average number of dorsal trunk interruptions per embryo includes those embryos with no interruptions. 
tor might partially obscure the normal pattern of BTL activity and cause a dominant phenotype. To test this hypothesis, we examined the effects of inducing $\lambda$ BTL in wild-type embryos. In stage 14 embryos, the dorsal trunk branches have fused normally (Fig. 3A); in stage $16 \mathrm{em}$ bryos, the basic embryonic tracheal system with the characteristic pattern of secondary branches is established (Fig. 3B). However, following expression of $\lambda \mathrm{BTL}$ at stage 11, development of primary branches was disrupted; the dorsal trunk was incompletely fused in most stage 14 embryos (Fig. 3C; Table 1) and abnormal primary branches and ectopic secondary branches were observed in most stage 16 embryos (Figs. 3D and 4).

To describe the effects of expressing $\lambda$ BTL on wildtype tracheal system development in more detail, several representative abnormalities are shown in Figure 4. Common abnormalities included the following: formation of three major cerebropharyngeal branches, instead of two (Fig. 4, cf. A and B); misguidance of cerebropharyngeal branches (Fig. 4B); extra branching of dorsal branches (Fig. 4, cf. C and D); absence of dorsal branches (Fig. 4D); misguidance and extra branching of ganglionic branches (Fig. 4, cf. E and F); absence of ganglionic branches (Fig. 4, cf. G and H); and failure of the lateral trunk branches from adjacent segments to fuse (Fig. 4H).

\section{Enhancement of $\lambda B T L$-induced tracheal cell migration defects by reduction in wild-type BTL}

The adverse effects of $\lambda \mathrm{BTL}$ on tracheal patterning could be attributable either to a lack of spatial regulation of the activity or to increased levels of BTL activity. If the de-
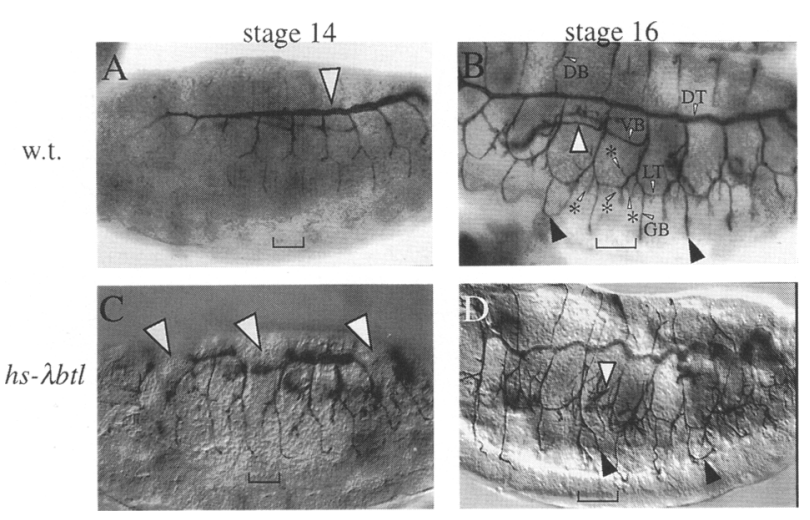

Figure 3. Effects of $\lambda$ BTL on the developing tracheal system in stage 14 and 16 embryos. Nomarski optics images of embryos stained for tracheal lumen antigen 2A12. Wild-type $(A, B)$ and $h s-\lambda b t l$ embryos in which expression of $h s-\lambda b t l$ had been induced at stage $11(C, D)$ are shown. $(A, C)$ Stage 14 embryos; $(B, D)$ stage 16 embryos. Normal $(A)$ and unfused $(C)$ dorsal trunks are indicated by the white arrowheads. A normal $(B)$ and an abnormal $(D)$ visceral branch are indicated by the white arrowheads. Other normal primary branches $(B)$ and their abnormal counterparts in $h s-\lambda b t l$ embryos $\langle D|$ are indicated by the black arrowheads. $(B)$ The following primary branches are indicated: $(\mathrm{DB})$ dorsal branch; (DT) dorsal trunk, (VB) visceral branch; (LT) lateral trunk; $(\mathrm{GB})$ ganglion branch. Asterisks indicate normal secondary branches. Bars, $25 \mu \mathrm{m}$.

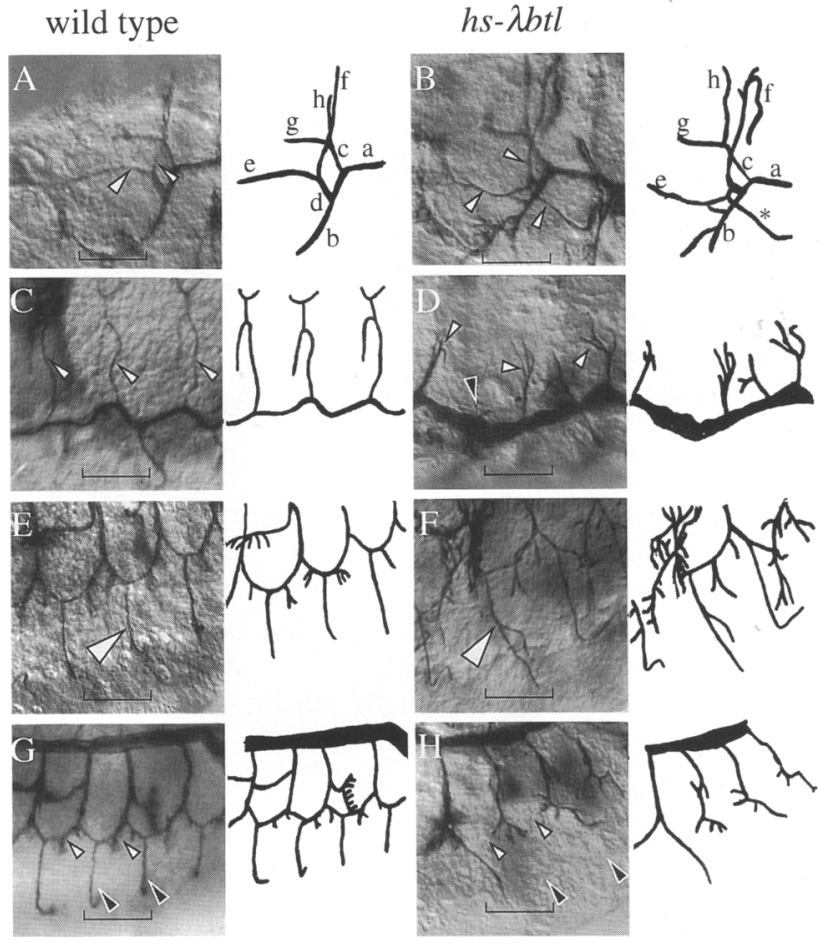

Figure 4. Representative abnormalities in the developing tracheal systems of $h s-\lambda b t l$ embryos. Nomarski optics images of embryos stained for tracheal lumen antigen 2A12. Wild-type $(A, C, E, G)$ embryos and embryos in which expression of $\lambda b t l$ was induced at stage $11(B, D, F, H)$ are shown. Embryos were fixed at stage 15. $(A, B)$ Arrowheads indicate the normal two cerebropharyngeal branches in $(A)$ and the three found in $(B) .(C, D)$ The arrowheads indicate normal $(C)$ and abnormal or missing $(D)$ dorsal branches. $(E)$ Normal ganglionic branches are indicated. $(F)$ The arrowhead indicates an ectopic branch emanating from one of the ganglionic branches. $(G, H)$ Fused lateral trunks (arrowheads) can be seen in $G$ whereas the lateral trunks are unfused in $H$. Schematic representations of the stained tracheal branches are shown next to each micrograph. $(A, B)$ The tracheal branches in the schematics are labeled with letters to facilitate identification of corresponding branches. $(B)$ The asterisk indicates the extra cerebropharyngeal branch.

fects observed in $\lambda b t l$ embryos were attributable to a lack of spatial regulation of BTL, one might predict that the defects would be exacerbated if endogenous, patterned BTL activity were reduced, for example, in flies heterozygous for loss-of-function mutations in the $b t l$ locus. On the other hand, if the $\lambda B T L$-induced defects were attributable to the high level of BTL activity, one might expect reducing the amount of endogenous receptor to reduce overall receptor activity levels and, therefore, to ameliorate the defects. If the levels of $\lambda B T L$ activity were so high as to overwhelm the endogenous activity completely, then one would expect reducing expression of the wild-type receptor to have no effect on the $\lambda B T L$-induced defects.

To distinguish between a requirement for spatial regulation of BTL activity and a requirement for quantitative regulation, we compared tracheal development in 
embryos that were wild-type for the endogenous $b t l$ locus with that of embryos that were heterozygous for $b t 1^{H 82 \Delta 3}$, a strong loss-of-function allele. After crossing $h s-\lambda b t l$ into the heterozygous $b t l^{H 82 \Delta 3}$ background, heat shock treatments were carried out. Stage-specific enhancement of $\lambda \mathrm{BTL}$-induced phenotypes was observed. For example, the dorsal trunk defects of stage $14 \mathrm{em}$ bryos were exaggerated in heterozygous $b t l^{H 82 \Delta 3} \mathrm{em}$ bryos (Fig. 5). Whereas induction of $\lambda B T L$ in otherwise wild-type embryos typically resulted in two to three interruptions in the dorsal trunk (Fig. 5A) and never more than four, induction of $\lambda \mathrm{BTL}$ in embryos heterozygous for $b t l^{H 82 \Delta 3}$ resulted in unfused dorsal trunks with four or more interruptions in about $40 \%$ of stage 14 embryos (Fig. 5B; Table 1).

To investigate further the interactions between endogenous BTL activity and $\lambda B T L$, we examined the effects of coexpression of $B T L^{\mathrm{K} \rightarrow R}$ and $\lambda B T L$. Expression of the kinase defective $\mathrm{BTL}^{\mathrm{K} \rightarrow \mathrm{R}}$ alone caused dominant-negative effects on tracheal development (Fig. 6, cf. A and B; see also Fig. $8 \mathrm{~B}$ ), presumably attributable to (1) binding of ligand to inactive $\mathrm{BTL}^{\mathrm{K} \rightarrow \mathrm{R}}$ dimers, reducing the effective ligand concentration, and (2) heterodimerization of inactive $\mathrm{BTL}^{\mathrm{K} \rightarrow \mathrm{R}}$ monomers with wild-type BTL protein. When the transgene was induced at stage 11 , defects in primary branching were observed, such as interruptions in the dorsal trunks and absence of some primary branches (Fig. 6B).

To address the effects of reducing endogenous BTL activity on the $\lambda B T L$-induced phenotypes, we coexpressed $\mathrm{BTL}^{\mathrm{K} \rightarrow \mathrm{R}}$ and $\lambda b t l$ at stage 11 . The effects of $\mathrm{BTL}^{\mathrm{K} \rightarrow \mathrm{R}}$ and $\lambda \mathrm{BTL}$ on primary branching appeared to be synergistic (Table 1). That is, primary branching was almost completely blocked in about $60 \%$ of embryos (Fig. 6D). Because formation of primary branches depends mainly on guided tracheal cell migrations, this observation indicated that the pattern of BTL activity was more important than the level of BTL activity during the period of cell migration.

\section{Effects of unregulated BTL activity on secondary and terminal tracheal branching}

It has been shown previously that $b t l$ is required for ex-

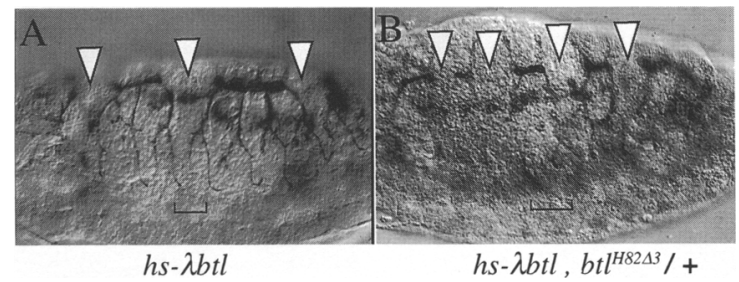

Figure 5. Effects of reducing $b t l$ gene copy number on $\lambda b t l$ phenotypes. Nomarski optics images of embryos stained for tracheal lumen antigen 2A12. Expression of $\lambda b t l$ was induced in either wild type $(A)$ or heterozygous $b t 1^{H 82 \Delta 3}(B)$ by delivering a heat shock at stage 11 ; then embryos were incubated at $18^{\circ} \mathrm{C}$ and fixed at stage 14. Genotypes were determined as described in Fig. 2. Defects in dorsal trunks are indicated.

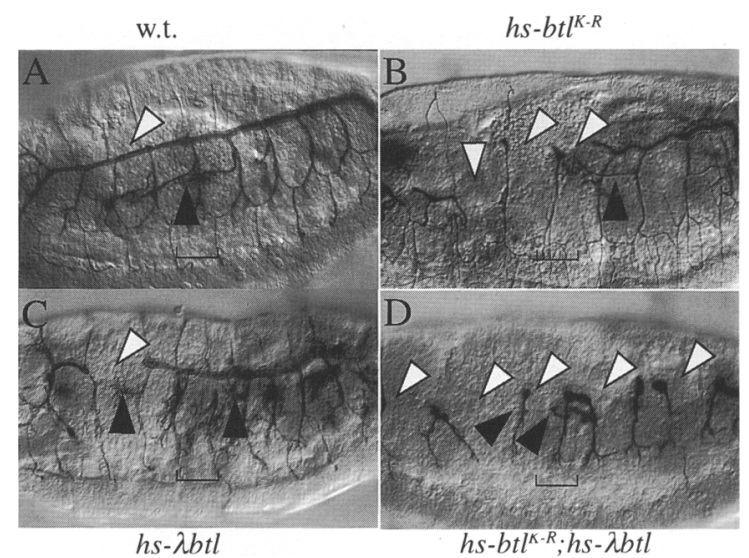

Figure 6. Effects of coexpressing $b t l^{K \rightarrow R}$ and $\lambda b t l$ at stage 11 . Nomarski optics images of embryos stained for tracheal lumen antigen 2A12. Expression of indicated transgenes induced at stage 11. Embryos were fixed at stage 15 . Compared with the wild-type tracheal system $|A|$, unfused dorsal trunks $\mid B, C, D$, white arrowheads) and abnormal visceral branches $(B, C, D$, black arrowheads) were obvious, among other abnormalities.

pression of secondary and terminal branch markers during tracheal development (Samakovlis et al. 1996). To confirm that the ectopic fine branches in $h s-\lambda b t l$ embryos arose because of a change in tracheal cell fate, we examined the expression of secondary and terminal branch markers in these embryos. As shown in Figure 7A, ectopic secondary branch formation was accompanied by the ectopic expression of the Pantip-1/pointed secondary branch marker. In these experiments, heat shocks were performed after stage 12 to reduce the probability that secondary branch abnormalities resulted as a secondary consequence of abnormal primary branching. The extra branching cells also expressed the terminal branch marker Terminal-1/DSRF (Fig. 7B). The extra branching cells presumably arose from a transformation in cell fate because, under some regimens of expression of $\lambda \mathrm{BTL}$, many or all tracheal cells in a primary branch can be induced to express the Terminal-1 marker (Fig. $7 \mathrm{C})$ even though the number of cells was unchanged.

\section{Rescue of $B T L^{\mathrm{K} \rightarrow \mathrm{R}}$-induced secondary branching defect by $\lambda B T L$ and suppression of $\lambda B T L$-induced ectopic secondary branching by $B T L^{\mathrm{K} \rightarrow \mathrm{R}}$}

The observation that BTL activity is both necessary and sufficient to induce secondary or terminal branches in tracheal cells implies that selective activation of BTL determines the normal pattern of secondary or terminal branches. To distinguish whether, within a given branch-forming cell, the level of BTL activity or the pattern of BTL activity was more important in determining secondary branches, we determined the effects of co-expressing $\mathrm{BTL}^{\mathrm{K} \rightarrow \mathrm{R}}$ and $\lambda \mathrm{BTL}$.

In contrast to the synergistic effects on primary branching, the effects of BTL ${ }^{\mathrm{K} \rightarrow \mathrm{R}}$ and $\lambda \mathrm{BTL}$ appeared to antagonize each other with respect to secondary branch- 


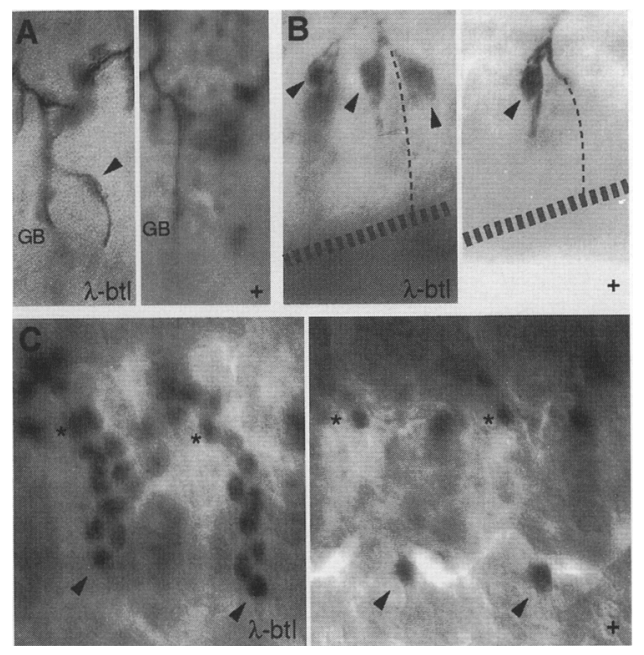

Figure 7. Expression of markers for secondary and terminal branch-forming cells in $h s-\lambda b t l$ embryos. $(A, B) h s-\lambda b t l$ embryos were heat shocked once at late stage 12 and once at late stage 13 , then aged and fixed and stained for $2 \mathrm{~A} 12$ antigen and the Pantip-1 (pointed) lacZ marker $(A)$ or the DSRF terminal branch marker $(B) .(A$, left $)$ The Pantip-1 marker can be seen in the cell nucleus of an ectopic secondary branch (arrowhead) that has formed in the middle of a ganglionic branch $(\mathrm{GB})$. $(A$, right $) \mathrm{A}$ wild-type embryo of the same age, for comparison, shows that the GB is unbranched and that the marker at this stage is not expressed in the GB except in the leading cell /not visible in this plane of focus). ( $B$, left $)$ DSRF protein is seen in the normal and two ectopic branches (arrowheads) that have formed at the end of the dorsal branch (position indicated by the thin dashed line). ( $B$, right) A wild-type embryo, for comparison, shows the single DSRF-expressing branch. The thick dashed line indicates the position of the dorsal trunk. $|C|$ Embryos were heat shocked late in stage 11 and again late in stage 12 and then aged and stained for DSRF at stage 13 or 14. Under these conditions (left), expression of DSRF is seen in most or all cells of the two GBs shown (arrowheads point to the leading cell of each GB). In a wild-type embryo of the same age (right), expression of DSRF is restricted to the leading cell (arrowheads). The asterisks indicate the position of a DSRF-expressing cell in the lateral trunk posterior branch from which the GB extends. In the $h s-\lambda b t l$ embryos, the number of cells in the GB is unchanged from wild type.

ing. When the $h s-b t l^{K \rightarrow R}$ transgene alone was induced at stage 12 , most primary branches remained unaffected but formation of secondary branches was inhibited. This effect was especially pronounced in the visceral region (Fig. 8B). In contrast, when both transgenes were induced at stage 12, most embryos looked more like wild-type than heat shock-treated embryos carrying either transgene alone (Fig. 8). These observations suggest that expression of $\lambda \mathrm{BTL}$ rescued formation of secondary branches in embryos expressing $\mathrm{BTL}^{\mathrm{K} \rightarrow \mathrm{R}}$ and expression of $h s-b t 1^{K \rightarrow R}$ inhibited induction of ectopic secondary branches because of expression of $\lambda b t l$. In contrast to the deleterious effects of $\lambda B T L$ on primary branching, constitutive BTL activity could replace endogenous BTL activity in formation of secondary branches.
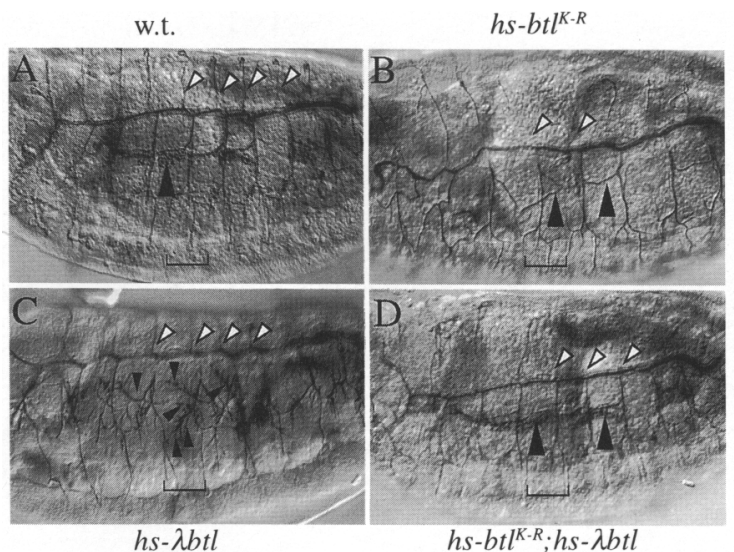

Figure 8. Effects of coexpressing $b t I^{K \rightarrow R}$ and $\lambda b t l$ at stage 12 on tracheal system development. Nomarski optics images of embryos stained for tracheal lumen antigen 2A12. (A) Wild-type stage- 15 tracheal system: $(B-D)$ Expression of indicated transgenes was induced at stage 12 , and embryos were fixed at stage 15. White arrowheads indicate dorsal branches, which can be scen in every segment of the tracheal system in $A$ and $C$ but are missing from the indicated segments in $B$ and $D$. Black arrowheads indicate visceral branches with fewer $(B)$ or more $(C)$ than the normal $|A|$ secondary branches.

\section{Effects of cell autonomous $\lambda$-BTL expression}

To determine whether the effects described so far were attributable primarily to cell autonomous effects of activated BTL, we used the GAL4/UAS system (Brand and Perrimon 1994) to direct $\lambda$ BTL expression specifically in tracheal cells. Germ-line transformants carrying a UAS$\lambda \mathrm{BTL}$ transgene were crossed to flies carrying a GAL4 enhancer trap line expressed in tracheal cells beginning in stage 13 (A. Brand, unpubl.). Embryos from such a cross were collected, aged, and stained for the $2 \mathrm{~A} 12$ tracheal lumen antigen. Stalled or missing primary branches as well as misguided and ectopic secondary branches were observed in nearly every embryo (Fig. 9). The defects occurred most frequently in the dorsal branches and the ganglionic branches, which form later than the dorsal trunk and lateral branches, perhaps as a result of the relatively late induction of $\lambda \mathrm{BTL}$.

\section{Effects of activated SEV on tracheal system development}

To determine the specificity of the effects of $\lambda B T L$, we examined the effects of expressing an activated SEV on tracheal system development. Compared with activated BTL, activated SEV induced at stage 11 did little harm to the developing tracheal system (Fig. 10A) despite strong deleterious effects on viability $170 \%$ of embryos arrested development following a 40-min heat shock, compared with $0 \%$ for $\lambda B T L$ embryos). Thus tracheal cell migration appeared to be specifically sensitive to changes in BTL activity. In contrast with the subtle effect on primary branching, ectopic SEV activity induced at stage 13 was capable of inducing numerous, ectopic secondary or 


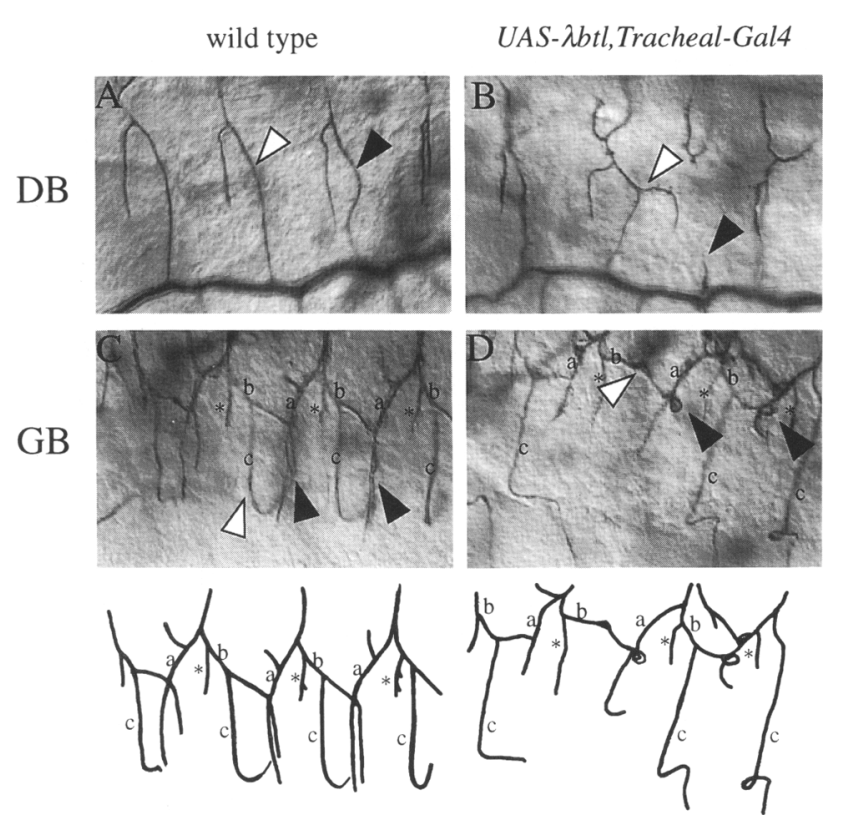

Figure 9. Effects of cell autonomous expression of $\lambda \mathrm{BTL}$. $(A)$ Wild-type dorsal branches are indicated by the arrowheads. $(B)$ In embryos expressing $\lambda$ BTL under the control of a tracheal GAL4 line, beginning at stage 13 , defective dorsal branches were observed in nearly every embryo. Ectopic secondary branches (white arrowhead) and stalled dorsal branches (black arrowhead) were commonly observed. $(C)$ In wild-type embryos, the ganglionic branch (white arrowhead, c) emanates from a lateral branch (b). Two secondary branches normally form (black arrowheads) at the point of fusion between lateral branches $(a, b)$ from adjacent segments. In embryos expressing $\lambda B T L$ under the control of tracheal GAL4 $(D)$, missing ganglionic branches were observed (white arrowhead) and misguided secondary branches were common (black arrowheads). Schematic representations of $|C|$ and $(D)$ are shown below. The asterisks indicate secondary branches that form normally, for a reference point.

terminal branches (Fig. 10B, and cf. D with C), which were considerably longer than those induced by $\lambda \mathrm{BTL}$. The differential effects of ectopic SEV activity on primary versus secondary or terminal branching further support the idea that BTL plays distinct roles in mediating different developmental processes to establish the complete tracheal system.

\section{Discussion}

The Drosophila tracheal system has become an informative model system for unraveling the molecular mechanisms underlying branching morphogenesis, a process that requires the coordination of cell fate determination and differentiation with directed cell migration, branch fusion, and tube formation. Although it has been shown previously that the BTL RTK is required for primary, secondary, and terminal branching in the tracheal system, our studies have addressed the question of whether spatially or quantitatively regulated BTL activity contributes to patterning tracheal cell migration and/or branching.

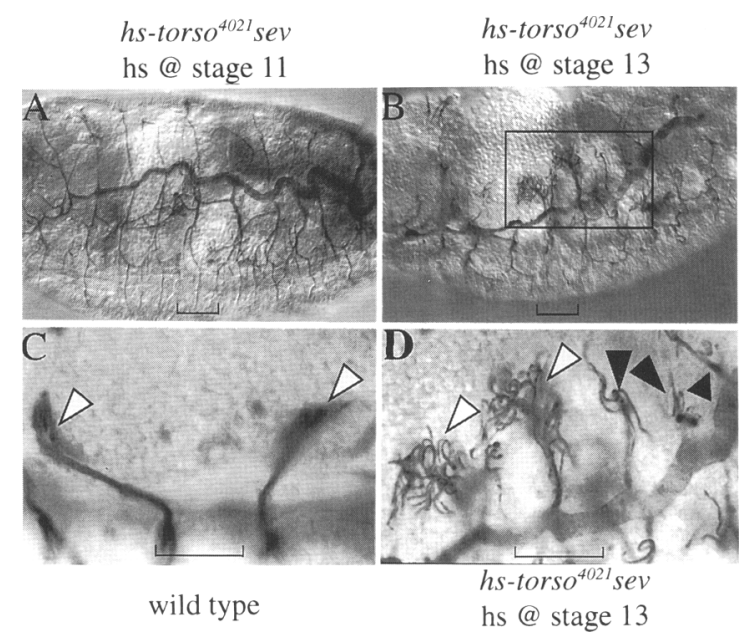

Figure 10. Effects of expressing activated SEV on tracheal system development. Nomarski optics images of embryos stained for tracheal lumen antigen 2A12. Embryos carrying the torso ${ }^{4021} \mathrm{sev}$ transgene under heat shock promoter control were heat shocked at either stage $11(A)$ or stage $13(B)$, aged, and then fixed at stage $15 .(A, B)$ The primary branching pattern looks normal. (D) Close-up view of the region indicated in $B$, showing multiple ectopic secondary branches emanating from visceral (white arrowheads) and dorsal (black arrowheads) branches, although these two kinds of branches are not normally found in the same focal plane. $(C)$ Comparable view of visceral branches in a wild-type embryo.

\section{Role of BTL in tracheal cell migration}

Although it has been known for some time that RTKs can mediate chemotaxis in vitro (Kundras et al. 1994), appreciation of the potential importance of a direct role for RTK signaling in cell and axon motility in vivo during development has been relatively recent. One of the first indications that RTKs might regulate cell migration came with the identification of c-Kit, which encodes an RTK, as the gene mutated in White mutant mice, which display cell migration defects (Fleischman 1993). More recently, mutations in other RTKs have been shown to lead to defects in neural crest cell migration (Schuchardt et al. 1994) and myoblast migration (Bladt et al. 1995) during mouse embryogenesis, as well as sex myoblast migration in the nematode (DeVore et al. 1995) and axon pathfinding in Drosophila (Callahan et al. 1995). Furthermore, RTK-mediated repulsion has been suggested to play a role in guiding retinal axons to the appropriate target sites in the tectum (Cheng et al. 1995; Drescher et al. 1995). Therefore the question of whether RTK activity can guide cell and axon migrations in vivo may have general implications.

The primary tracheal branches of the Drosophila embryo form when individual cells, or pairs of cells, located at six different positions within each cluster of about 80 tracheal cells, lead the directed migration along stereotypical pathways to form the six primary branches. This phase of tracheal development clearly requires the activity of the BTL RTK because it does not occur in $b t l$ 
mutant embryos. One possibility is that BTL, and perhaps other RTKs, guides cell migration directly via a chemotactic mechanism. This model predicts that the spatial pattern of BTL activity would be critical to primary tracheal branching. The inability of constitutively active BTL to rescue $b t l$ mutant defects supports the chemotactic guidance model.

Our results indicate that the defects caused by $\lambda B T L$ were not primarily attributable to extraordinarily high levels of receptor activity because decreasing endogenous $b t l$ expression or activity enhanced $\lambda B T L$ tracheal cell migration defects. If the level of $\lambda \mathrm{BTL}$ activity were much higher than the endogenous level, we would have expected reducing the level of endogenous activity to have little or no effect. Moreover, if the overall level of BTL activity were the critical factor in tracheal cell migration, reducing the endogenous activity should have ameliorated the $\lambda B T L$ migration defects. Nor does temporal control of BTL activity seem to be particularly important, as we have observed rescue of btl mutant embryos following heat shock-induced expression of wildtype BTL as late as $9 \mathrm{hr}$ after egg laying (stage 12 or 13; T. Lee and D.J. Montell, unpubl.). Taken together, the results support the notion that spatial regulation of BTL activity contributes to the guidance of tracheal cell migration.

It is likely that the tracheal system defects we observed were attributable primarily to cell autonomous effects because tracheal cell-specific expression of $\lambda B T L$ caused qualitatively similar defects. Missing or stalled primary branches were observed among the dorsal and ganglionic branches, and misguided or ectopic secondary branches were common. Similar defects were not observed in the dorsal trunk or in lateral branches in this genotype, although they did occur in the $h s-\lambda b t l \mathrm{em}$ bryos. The differences were presumably attributable to differences in the timing of expression of activated BTL induced by the tracheal GAL4 comparcd with heat shock, although we cannot rule out some effect of expression level.

The hypothesis that BTL may act as a chemotactic guidance receptor is further supported by the recent discovery of a ligand for BTL, the product of the branchless (bnl) gene (Sutherland et al. 1996). bnl mutations cause tracheal defects that are strikingly similar to $b t l$ defects. Furthermore, the $b n l$ gene encodes a protein with some sequence similarity to FGFs and is expressed in the dynamic, spatially restricted pattern consistent with a role in guidance. In addition, a heat shock bnl transgene increases the level of BTL tyrosine phosphorylation, suggesting that BNL can activate BTL. The spatial distribution of bnl indicates that BTL activity is normally spatially regulated, whereas our results show that such regulation is essential to normal tracheal patterning.

The disruption of tracheal cell migration by $\lambda$ BTL reported here contrasts with the effects of a previously reported, constitutively active BTL receptor, Tor ${ }^{4021}$. BTL. Tor ${ }^{4021}$-BTL does not cause dominant tracheal defects. Furthermore, Tor ${ }^{4021}$-BTL can provide partial rescue of tracheal cell migration in btl mutants, in the dor- sal trunk and some lateral branches (Reichman-Fried et al. 1994). We also observed dorsal trunk fusion in a minority of segments following expression of hs- $\lambda$ BTL, but this occurred much later in development than normal. It may be that some tracheal cells, such as those forming the dorsal trunk and possibly lateral branches, are slightly less dependent on spatially localized BTL activity for their directed migration, compared with the cells forming dorsal, visceral, or ganglionic branches. Thus, although constitutive BTL activity can rescue some branches, in a fraction of btl mutant embryos, regulated BTL activity is clearly necessary for tracheal cell migration to be complete, reproducible, accurate, and timely.

The inability of Tor ${ }^{4021}$-BTL to cause dominant defects may be attributable to its low level of activity. We have shown that Tor ${ }^{4021}$-BTL is phosphorylated on tyrosine only to low levels because more than half of the Tor ${ }^{4021}$-BTL protein was not detectably tyrosine phosphorylated. The Tor ${ }^{4021}$ extracellular domain appears to confer varying degrees of ligand-independent activity, depending on the kinase domain to which it is fused. Although a Tor ${ }^{4021}$-SEV receptor and Tor ${ }^{4021}$ itself do seem to be constitutively active and biologically potent, a Tor ${ }^{4021}$-TORPEDO chimera did not display the phenotypes expected of a constitutively activated receptor ( $\mathrm{T}$. Schupbach, pers. comm.|. It seems likely, then, that the level of Tor ${ }^{021}$-BTL activity is lower than the level of endogenous, patterned activity, and so does not obscure the normal pattern of BTL activity and does not cause dominant defects.

\section{Role of BTL in secondary and terminal tracheal branching}

Unlike primary tracheal branching, which involves the coordinated rearrangement and migration of cells, formation of secondary and terminal branches are unicellular processes (Samakovlis et al. 1996). Our results indicate that BTL activity may play a different role in secondary and terminal tracheal branching than in primary branching. The normal pattern of secondary branching apparently depends on the restriction of high levels of BTL activity to a small number of cells, because ectopic secondary and terminal branches were observed in embryos with heat shock-induced expression of $\lambda \mathrm{BTL}$. However, the observation that reducing endogenous $b t l$ ameliorated the phenotype suggests that the ectopic branches were attributable to higher than normal levels of BTL activity in cells that do not normally form secondary branches.

Distinct roles for BTL activity in tracheal cell migration versus branching are also supported by the observation that changes in gene expression are required for tracheal cells to produce secondary and/or terminal branches, whereas there is no evidence that tracheal cell migration in response to BTL is mediated through nuclear signaling. 
Lee et al.

\section{Specificity of BTL RTK signaling in tracheal system development}

Although several RTKs have been implicated in mediating cell migration (Schuchardt et al. 1994; Bladt et al. 1995; DeVore et al. 1995), the roles of known downstream effectors for RTKs in cell migration remain unclear. Most well-studied RTKs appear to share some common downstream effectors and have similar biological activities so that one RTK can partially replace another RTK (Dikic et al. 1994). One example is that many RTKs activate the Ras-Raf-MAP kinase pathway (Brunner et al. 1994; Johnson and Vaillancourt 1994). However, the signaling pathway mediating RTK-dependent cell migration is poorly understood. The observation that activated SEV, unlike activated BTL, had no significant effect on tracheal cell migration suggests the involvement of downstream effectors specific to the migration response.

In contrast with the specificity of BTL in tracheal cell migration, activated SEV appeared to have a similar effect as activated BTL in producing ectopic secondary of terminal branch-forming cells. Thus common downstream effectors, shared by BTL and SEV, might mediate cell fate determination in the tracheal system.

In summary, the effects of constitutive BTL activity on tracheal development suggest that patterned activation of the BTL receptor is required for tracheal cell migration during primary branch formation as well as for proper patterning of secondary and terminal cell fates. Although our data support the hypothesis that BTL could play a direct role in guidance of tracheal cell migration, demonstration of chemotactic behavior of tracheal cells in response to BNL or another ligand would be definitive.

\section{Materials and methods}

DNA constructs

One pair of primers $\left(\lambda 5^{\prime}\right.$-ATTGCGGCCGCCCATGGTTACCTGGAGG and $\lambda 3^{\prime}$-ATTAGATCTCCAGTTGTAAAGGGGAGG) was used to amplify the sequence encoding the dimerization domain of bacteriophage $\lambda \mathrm{cI}$ repressor from the plasmid pCIXR (a gift from Enrique Amaya, University of California, Berkeley) containing a constitutively active Xenopus FGF receptor. After digestion with NotI and BgIII, the PCR-amplified product was subcloned into pBS containing the $b t l$ gene. With linker tailing, another NotI site was generated on the EcoRI site located on the $3^{\prime}$ end of the BTL open reading frame. Then the NotI fragment containing the $\mathrm{BBTL}$ sequence was subcloned into the plasmid pCasper-hs.

A standard site-specific mutagenesis (Kunkel et al. 1987) was performed to mutate the conserved lysine residue within the ATP binding domain to arginine with the oligonucleotide (ATCGTGGCCGTCCGGATGGTCAAGGAG). Then the $X m n I-X h o l$ fragment containing the $\mathrm{K} \rightarrow \mathrm{R}$ mutation was subcloned into the kanamycin-resistant vector $\mathrm{pHXK}$ containing the $b t l$ gene. Finally, the mutated $b t l$ gene was subcloned into pCasper-hs with NotI as the cloning site.

Fly strains and germ-line transformation

Standard procedures were used for culturing flies and for P ele- ment-mediated germ-line transformation (Rubin and Spradling 1982). $w^{1118}$ was used for P element-mediated germ-line transformation. $b t 1^{H 82 \Delta 3} / \mathrm{Tm} 3$ is a fly strain heterozygous for a strong loss-of-function btl allele (Klambt et al. 1992). hs-btl is a transgenic fly strain carrying a wild-type $b t l$ gene under heat shock promoter control (Murphy et al. 1995). hs- $\lambda b t l$ is a transgenic fly strain carrying the $\lambda b t l$ fusion gene under heat shock promoter control, in the pCaSpeR-hs vector /a gift from C. Thummel, University of Utah, Salt Lake City). $h s-b t l^{K \rightarrow R}$ is a transgenic fly strain carrying the $b t t^{K \rightarrow R}$ gene in the pCaSpeR-hs vector. Recombinant chromosomes containing either $h s-\lambda b t l$ or $h s-b t l^{K \rightarrow R}$ with $b t I^{H 8243}$ were constructed. The $U A S-\lambda b t l$ constructs were generated by cloning the $b t l$ cDNA fragment into the pUAST vector (Brand and Perrimon 1994). The tracheal GAL4 line used in this study was kindly provided by A. Brand (Cambridge University, UK).

\section{Heat shock treatment}

Egg collection plates containing embryos were placed into petri dishes and sealed with plastic wrap to prevent desiccation. Dishes were incubated at $37^{\circ} \mathrm{C}$ for $40 \mathrm{~min}$ to induce expression of transgenes under heat shock promoter control.

\section{Immunoprecipitation and immunoblotting}

Embryos were dechorionated with $50 \%$ bleach for $10 \mathrm{~min}$; then, $\sim 100 \mu \mathrm{l}$ packed embryos were homogenized in $1 \mathrm{ml}$ of RIPA buffer $11 \%$ Noniodet P- $40,0.5 \%$ sodium deoxycholate, $0.1 \%$ SDS, $20 \mathrm{~mm}$ Tris at $\mathrm{pH} 8,137 \mathrm{~mm} \mathrm{NaCl}, 10 \%$ glycerol, and $2 \mathrm{~mm}$ EDTA) containing $100 \mu \mathrm{g} / \mathrm{ml} \mathrm{PMSF}$ at $4^{\circ} \mathrm{C}$. After incubation on ice for $15 \mathrm{~min}$, the homogenate was centrifuged at $12,000 \mathrm{~g}$ for $10 \mathrm{~min}$. Sodium orthovanidate was added to the supernatant to a final concentration of $0.2 \mathrm{~mm}$. Then the embryo lysate was incubated with either $8 \mu \mathrm{l} \alpha$-pY (Upstate Biotechnology \#05321 ) or $8 \mu \mathrm{l} \alpha$-BTL polyclonal mouse antiserum (collected from $\mathrm{balb} / \mathrm{c}$ mice, which had been injected three times, two weeks apart, with gel-purified, His-tagged fusion protein containing the cytoplasmic portion of BTL) at $4^{\circ} \mathrm{C}$ overnight. The immune complex was precipitated with goat antimouse IgG-agarose (Sigma \#A-6531). After washing the agarose with RIPA buffer three times, it was resuspended in $100 \mu$ l Laemmli sample buffer. Prior to electrophoresis, samples were boiled for $5 \mathrm{~min}$. After SDS-PAGE, protein was transferred to the nitrocellulose membrane. Then the membrane was blocked with $4 \%$ milk in PBS for $1 \mathrm{hr}$, incubated with primary Ab in PBT $(1 \times \mathrm{PBS}, 0.1 \%$ tween-20, and $0.2 \% \mathrm{BSA}$ ) at $4^{\circ} \mathrm{C}$ overnight, washed with PBS containing $0.1 \%$ tween- 20 three times for $10 \mathrm{~min}$ each, incubated with peroxidase-conjugated secondary Ab in PBT for one hour at room temperature, washed with PBS containing $0.1 \%$ tween-20 three times for $10 \mathrm{~min}$ each, and finally developed in PBS containing $0.05 \%$ sodium diaminobenzidine and $0.03 \%$ hydrogen peroxide.

\section{Immunohistochemistry}

Embryos were collected for $1 \mathrm{hr}$ on grape juice agar plates, treated as indicated in the figure legends, and then fixed at specific stages. Fixation of embryos was carried out as follows. After dechorionation with $50 \%$ bleach for $10 \mathrm{~min}$, embryos were transferred into fixation buffer consisting of $900 \mu \mathrm{l}$ PEM (0.1 $\mathrm{M}$ Pipes, $2 \mathrm{~mm}$ EGTA, and $1 \mathrm{~mm} \mathrm{Mg}_{2} \mathrm{SO}_{4}$ with final $\mathrm{pH}$ 6.95), $100 \mu 137 \%$ formaldehyde, and $300 \mu l n$-heptane. After shaking at room temperature for $30 \mathrm{~min}$, embryos were washed with methanol five times, and then with PBT three times. After incubation in PBT for 30 min, embryos were then incubated in 
PBT containing $2 \mathrm{~A} 12$ monoclonal $\mathrm{Ab}(1: 1$ dilution $)$ at $4^{\circ} \mathrm{C}$ overnight. Following five washes with PBT over the course of 2 $\mathrm{hr}$, embryos were incubated in PBT containing biotinylated goat antimouse IgM (Vector BA-2020) for $2 \mathrm{hr}$ at room temperature. Following five washes with $\mathrm{PBT}$, embryos were developed with the Vectastain ABC kit (Vector Labs, Burlingame, CA). The Pantip-1 marker and the anti-DSRF antisera are described elsewhere (Guillemin et al. 1996; Samakovlis et al. 1996).

\section{Acknowledgments}

This work was supported by grant GM46425 from the National Institutes of Health and by a Lucille P. Markey Scholar Award to D.J.M.

The publication costs of this article were defrayed in part by payment of page charges. This article must therefore be hereby marked "advertisement" in accordance with 18 USC section 1734 solely to indicate this fact.

\section{References}

Basler, K., B. Christen, and E. Hafen. 1991. Ligand-independent activation of the sevenless receptor tyrosine kinase changes the fate of cells in the developing Drosophila eye. Cell 64: 1069-1081.

Bladt, F., D. Riethmacher, S. Isenmann, A. Aguzzi, and C. Birchmeier. 1995. Essential role for the c-met receptor in the migration of myogenic precursor cells into the limb bud. $\mathrm{Na}$ ture 376: 768-771.

Brand, A.H. and Perrimon, N. 1994. Raf acts downstream of the EGF receptor to determine dorsoventral polarity during Drosophila oogenesis. Genes \& Dev. 8: 629-639.

Brunner, D., N. Oellers, J. Szabad, W.H. Biggs III, S.L. Zipursky, and E. Hafen. 1994. A gain-of-function mutation in Drosophila MAP kinase activates multiple receptor tyrosine kinase signaling pathways. Cell 76: 875-888.

Callahan, C.A., M.G. Muralldhar, S.E. Lundgren, A.L. Scully, and J.B. Thomas. 1995. Control of neuronal pathway selection by a Drosophila receptor protein-tyrosine kinase family member. Nature 376: 171-174.

Cheng, H-J., M. Nakamoto, A.D. Bergemann, and J.G. Flanagan. 1995. Complementary gradients in expression and binding of ELF- 1 and Mek4 in development of the topographic retinotectal projection map. Cell 82: 371-382.

DeVore, D.L., H.R. Horvitz, and M.J. Stern. 1995. An FGF receptor signaling pathway is required for the normal cell migrations of the sex myoblasts in C. elegans hermaphrodites. Cell 83: 611-620.

Dickson, B., F. Sprenger, and E. Hafen. 1996. Prepattern in the developing Drosophila eye revealed by an activated torsosevenless chimeric receptor. Genes \& Dev. 6: 2327-2339.

Dikic, I., J. Schlessinger, and I. Lax. 1994. PC12 cells overexpressing the insulin receptor undergo insulin-dependent neuronal differentiation. Curr. Biol. 4: 702-708.

Drescher, U., C. Kremoser, C. Handwerker, J. Loschinger, M. Noda, and F. Bonhoeffer. 1995. In vitro guidance of retinal ganglion cell axons by RAGS, a $25 \mathrm{kDa}$ tectal protein related to ligands for Eph receptor tyrosine kinases. Cell 82: 359370.

Fantl, W.J., D.E. Johnson, and L.T. Williams. 1993. Signalling by receptor tyrosine kinases. Annu. Rev. Biochem. 62: 453481.

Fleischman, R.A. 1993. From white spots to stem cells: the role of the Kit receptor in mammalian development. Trends Genet. 9: 285-290.
Glazer, L. and B.-Z. Shilo. 1991. The Drosophila FGF receptor homolog is expressed in the embryonic tracheal system and appears to be required for directed trachael cell extension. Genes \& Dev. 5: 697-705.

Guillemin, K., J. Groppe, K. Ducker, R. Treisman, E. Hafen, M. Affolter, and M.A. Krasnow. 1996. The pruned gene encodes the Drosophila serum response factor and regulates cytoplasmic outgrowth during terminal branching of the tracheal system. Development 122: 1353-1362.

Johnson, G.L. and R.R. Vaillancourt. 1994. Sequential protein kinase reactions controlling cell growth and differentiation. Curr. Biol. 6: 230-238.

Klambt, C., L. Glazer, and B.Z. Shilo. 1992. breathless, a Drosophila FGF receptor homolog, is essential for migration of tracheal and specific midline glial cells. Genes \& Dev. 6: $1668-1678$.

Kundras, V., J.A. Escobedo, A. Kazlauskas, H.K. Kim, S.G. Rhee, L.T. Williams, and B.R. Zetter. 1994. Regulation of chemotaxis by the platelet-derived growth factor receptor-beta. $\mathrm{Na}$ ture 367: 474-476.

Kunkel, T.A., J.D. Roberts, and R.A. Zakour. 1987. Rapid and efficient site-specific mutagenesis without phenotypic selection. Methods Enzymol. 154: 367-382.

Manning, G. and M.A. Krasnow. 1993. Development of the Drosophila tracheal system. In The development of Drosophila melanogaster. (ed. M. Bate, and A.M. Arias), pp. 609685. Cold Spring Harbor Laboratory Press, Cold Spring Harbor, NY.

Montell, D.J. 1994. Moving right along: Regulation of cell migration during Drosophila development. Trends Genet. 10: $59-62$.

Murphy, A.M., T. Lee, C.M. Andrews, B.Z. Shilo, and D.J. Montell. 1995. The Breathless FGF receptor homolog, a downstream target of Drosophila C/EBP in the developmental control of cell migration. Development 121: 2255-2263.

Obermeier, A., R.A. Bradshaw, K. Seedorf, A. Choidas, and J. Schlessinger. 1994. Neuronal differentiation signals are controlled by nerve growth factor receptor/Trk binding sites for SHC and PLCy. EMBO J. 13: 1585-1590.

Reichman-Fried, M. and B.-Z. Shilo. 1995. Breathless, a Drosophila FGF receptor homolog, is required for the onset of tracheal cell migration and tracheole formation. Mechanism of Development 52: 265-273.

Reichman-Fried, M., B. Dickson, E. Hafen, and B.Z. Shilo. 1994. Elucidation of the role of breathless, Drosophila FGF receptor homolog, in tracheal cell migration. Genes \& Dev. 8: $428-439$.

Rubin, G.M. and A.C. Spradling. 1982. Genetic transformation of Drosophila with transposable element vectors. Science 218: 348-353

Samakovlis, C., N. Hacohen, G. Manning, D. Sutherland, K. Guillemin, and M.A. Krasnow. 1996. Branching morphogenesis of the Drosophila tracheal system occurs by a series of morphologically distinct but genetically coupled branching events. Development 122: 1395-1407.

Schuchardt, A., V. D'Agati, L. Larsson-Blomberg, F. Costantini, and V. Pachnis. 1994a. Defects in the kidney and enteric nervous system of mice lacking the tyrosine kinase receptor Ret. Nature 367: 380-383.

Sutherland, D., C. Samakovlis, and M.A. Krasnow. 1996. branchless encodes a Drosophila fibroblast growth factor homolog that controls tracheal cell migration and branching. Cell (in press). 


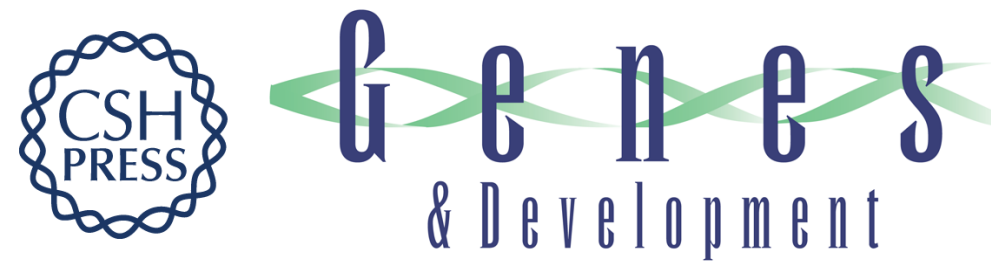

\section{Regulated Breathless receptor tyrosine kinase activity required to pattern cell migration and branching in the Drosophila tracheal system.}

T Lee, $\mathrm{N}$ Hacohen, M Krasnow, et al.

Genes Dev. 1996, 10:

Access the most recent version at doi:10.1101/gad.10.22.2912

References This article cites 26 articles, 8 of which can be accessed free at:

http://genesdev.cshlp.org/content/10/22/2912.full.html\#ref-list-1

License

Email Alerting Service

Receive free email alerts when new articles cite this article - sign up in the box at the top right corner of the article or click here.

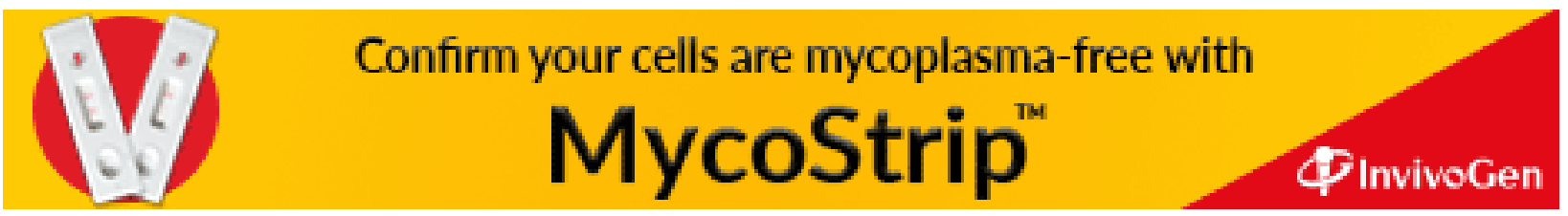

